\title{
Association of Diabetes and Severe COVID-19 Outcomes: A Rapid Review and Meta-Analysis
}

\author{
Mmamapudi Kubjane ${ }^{\mathrm{a}}$, Nicole McCreedy ${ }^{\mathrm{b}, \mathrm{c}, \mathrm{i}}$, Bertrand Cariou ${ }^{\mathrm{d}}$, \\ Miguel A. Rubio ${ }^{\mathrm{e}}$, Ulrik Haagen Panton ${ }^{\mathrm{f}}$, Christian Hvid, \\ Nadilka Hettiarachchige ${ }^{\mathrm{f}}$, Helen Mearns ${ }^{\mathrm{c}, \mathrm{g}, \mathrm{h}}$
}

\begin{abstract}
Background: Addressing the urgent need for evidence on diabetes as a serious comorbidity for severe illness and death from coronavirus disease 2019 (COVID-19), we investigated the association between diabetes and COVID-19 disease severity in patients hospitalized due to COVID-19.

Methods: This rapid review and meta-analysis was undertaken in adherence with the Preferred Reporting Items for Systematic Reviews and Meta-Analysis (PRISMA) guidelines. MEDLINE and EMBASE were searched for studies published between January 1 and May 20, 2020. Studies included were English language, peer-reviewed, observational studies of adults hospitalized for COVID-19 with reported clinical course and living with or without diabetes. The severity of clinical course was assessed using a composite outcome (mortality, admittance to intensive care unit (ICU), requirement for invasive mechanical ventilation (IMV), clinically defined severe or critical disease). Data and adjusted measures of association were extracted from published reports, and meta-analysis was performed using a random effects model. The protocol was registered with OSF (https://osf.io/agsyb/).
\end{abstract}

Results: A literature search yielded 431 articles, of which 45 studies

Manuscript submitted September 20, 2020, accepted October 3, 2020

Published online October 16, 2020

${ }^{a}$ Centre for Infectious Disease Epidemiology and Research, School of Public Health and Family Medicine, University of Cape Town, Cape Town, South Africa

${ }^{b}$ Last Mile P/S, Copenhagen, Denmark

'School of Public Health and Family Medicine, University of Cape Town, Cape Town, South Africa

d'institut du thorax, UNIV Nantes, CHU Nantes, Inserm, CNRS, Nantes, France

${ }^{\mathrm{e} E n d o c r i n o l o g y}$ and Nutrition Department, Hospital Clinico San Carlos, IDISSC, Complutense University, Madrid, Spain

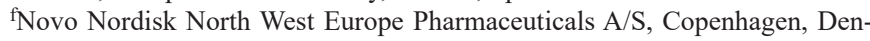
mark

gVaccines for Africa Initiative, University of Cape Town, Cape Town, South Africa

hIndependent Consultant, Hamilton, Waikato, New Zealand

${ }^{\mathrm{i} C}$ Corresponding author: Nicole McCreedy, School of Public Health and Family

Medicine, University of Cape Town, Cape Town, South Africa.

Email: nm@last-mile.dk

doi: https://doi.org/10.14740/jem698
(22,091 patients) met the inclusion criteria and 14 studies $(12,383$ patients) reported an adjusted measure of association for diabetes with the composite outcome with pooled hazard ratio 1.59 (95\% confidence interval $\left.1.3-1.93 ; \mathrm{I}^{2}=0 \%, \mathrm{P}=0.820\right)$ and pooled odds ratio of 2.15 (95\% confidence interval $\left.1.63-2.83 ; \mathrm{I}^{2}=0 \%, \mathrm{P}=0.892\right)$; evidence by GRADE was moderate.

Conclusions: People living with diabetes are more likely to develop severe COVID-19 clinical course if hospitalized for COVID-19 than people not living with diabetes. To inform clinical decision-making during the pandemic, our findings support that people living with diabetes who are hospitalized for COVID-19 should be prioritized when triaged as at increased risk for the development of severe clinical course.

Keywords: Diabetes; COVID-19; Severe COVID-19 outcomes; Meta-analysis

\section{Introduction}

The highly contagious coronavirus disease 2019 (COVID-19) was first reported in Wuhan, China, in December 2019. By March 11, 2020 the World Health Organization (WHO) had declared the outbreak a pandemic [1]. COVID-19 which typically presents with flu-like symptoms such as fever, cough, breathing difficulties, tiredness, and muscle aches, continues to spread worldwide at an alarming rate, with devastating impacts for individuals, healthcare systems, and economies [2]. The majority of infections result in mild disease, while approximately $20 \%$ of individuals develop severe forms of the disease [3]. Severe and critical disease in patients with COVID-19 is characterised by the need for specialized treatment in intensive care units (ICUs), often requiring invasive mechanical ventilation (IMV), and may result in death or long-term negative health effects, as was seen in previous coronavirus infections [4]. People most at risk for becoming seriously ill are the elderly and those with comorbidities such as diabetes, obesity, or hypertension $[5,6]$.

The International Diabetes Federation estimates that 463 million adults aged 20 - 79 were living with diabetes in 2019, equalling a global prevalence of $9.3 \%$ [7]. Diabetes is a chronic condition often associated with several serious complications, 
which if not managed appropriately, can decrease a person's quality of life or even lead to premature death [7]. People living with diabetes have also been shown to have worse clinical outcomes when infected with a range of infectious pathogens, highlighting their potential vulnerability in the event of a viral outbreak such as COVID-19 [8]. This fact is supported by emerging evidence from the current pandemic, where the most prevalent comorbidities reported in patients hospitalized due to COVID-19 are hypertension 32\% (95\% confidence interval (CI) 31 - 33), diabetes $22 \%$ (95\% CI 21 - 23), heart disease $13 \%$ (95\% CI 13 - 14), and chronic obstructive pulmonary disease (COPD) 8\% (95\% CI 7 - 8) [9]. A meta-analysis of 33 studies by Kumar et al (2020) [10] also identified an association between diabetes and severe COVID-19 in patients with diabetes hospitalized due to COVID-19, as well as a significantly increased risk of mortality, compared to patients without diabetes.

Given the current scale of the pandemic and the possible consequences for the many people living with diabetes, there is an urgent need to generate evidence rapidly to support healthcare professionals and decision-makers. This study seeks to address this by building on the current growing body of knowledge and providing up-to-date information for evidence-based clinical decision-making and planning for healthcare provision for those most at risk. The primary objective is to assess the association between diabetes and severe COVID-19 clinical course in patients hospitalized due to COVID-19. The secondary objective is to assess the prevalence of diabetes in patients hospitalized due to COVID-19. The exploratory objective is to report putative prognostic factors as identified in studies with hospitalized patients living with diabetes who developed severe COVID-19 clinical course.

\section{Materials and Methods}

\section{Search strategy and selection criteria}

This rapid review and meta-analysis was undertaken in adherence with the Preferred Reporting Items for Systematic Reviews and Meta-Analysis (PRISMA) guidelines [11]. MEDLINE and EMBASE were searched between January 1 and May 20, 2020. Keywords and MeSH terms specific to each database were used in the search, and COVID-19 search terms were based on the OVID Expert Search Team's validated COVID-19 search string [12]. Detailed search strategies are included in here (Supplementary Material 1, www.jofem.org). Identified articles and previous reviews were snowballed for articles that may have included data useful to this study.

Full texts were reviewed by one of five reviewers, with excluded studies verified independently by a second reviewer. Disagreements were resolved by consensus. Reasons for exclusion were recorded for all studies excluded after a fulltext review and reported in the PRISMA diagram. Abstracts, conference proceedings, letters, and other non-peer-reviewed studies were excluded. Only peer-reviewed, published studies (including accepted manuscripts in press) were included. Non-English language studies were excluded. Authors were not contacted for missing data.

Observational cohort and case-control studies that compare exposure with outcomes between different groups [13] were included to achieve the primary and secondary objectives, and any relevant information pertaining to the exploratory objective was reported. The study population included children, adolescents, and adults who were hospitalized due to suspected, probable, or confirmed COVID-19. To be eligible for inclusion, studies must have compared outcomes in a group of exposed individuals (people with diabetes) with a group of unexposed individuals (people without diabetes).

\section{Data extraction and synthesis}

The level of data sought, for the primary objective and for inclusion in the meta-analysis, was summary estimates of adjusted measure of association of diabetes with the composite outcome of COVID-19. The composite outcome was any of the following: mortality, admittance to ICU, requirement for IMV, or clinically diagnosed with refractory, progressive, severe, or critical disease according to one of the standard predefined criteria of the WHO [14]; or National Health Commission of China (version 3 - 5) [15]; or American Thoracic Society guidelines [16]. The level of data sought, for the secondary objective and included in the meta-analysis, was individual patient-level data of the proportion of people with diabetes in hospitalized COVID-19 patients with composite outcome. The level of data sought, for the exploratory objective, was summary estimates of association of putative prognostic factors with diabetes and the composite outcome of COVID-19. The protocol is available online at https://osf.io/agsyb/.

Data were extracted by one reviewer and checked by a second reviewer onto a custom spreadsheet, including participant characteristics, exposure and comparator characteristics, and outcomes of interest. No duplicate data were found. Possible study population overlap was investigated, with the study author contacted twice but with no response, the potentially overlapping studies were reported here (Supplementary Material 1, www.jofem.org), and an exploratory meta-analysis was performed with these studies excluded with results reported (Supplementary Material 1, www.jofem.org). The primary objective used the DerSimonian and Laird random effects model to report the adjusted measures of effect (hazard, risk, odds ratio) across the different studies with the $95 \%$ CI [17]. The secondary objective used the DerSimonian and Laird random effects model to pool the extracted crude proportions from different studies and report prevalence with a $95 \%$ CI. The $\mathrm{I}^{2}$ statistic was used to assess the level of heterogeneity between outcomes from different studies for the primary objective. $\mathrm{I}^{2}>50 \%$ was predefined as a high level of heterogeneity between studies. A sub-group analysis was to be performed if heterogeneity was found. The predefined sub-group analysis was by outcome, exposure, geographical region, and study quality. A post hoc analysis by median age was included. A risk of bias assessment with the NewcastleOttawa Scale (NOS) [18] was independently conducted by two reviewers, compared, and consensus was reached. The primary outcome was assessed with GRADE. A funnel plot 


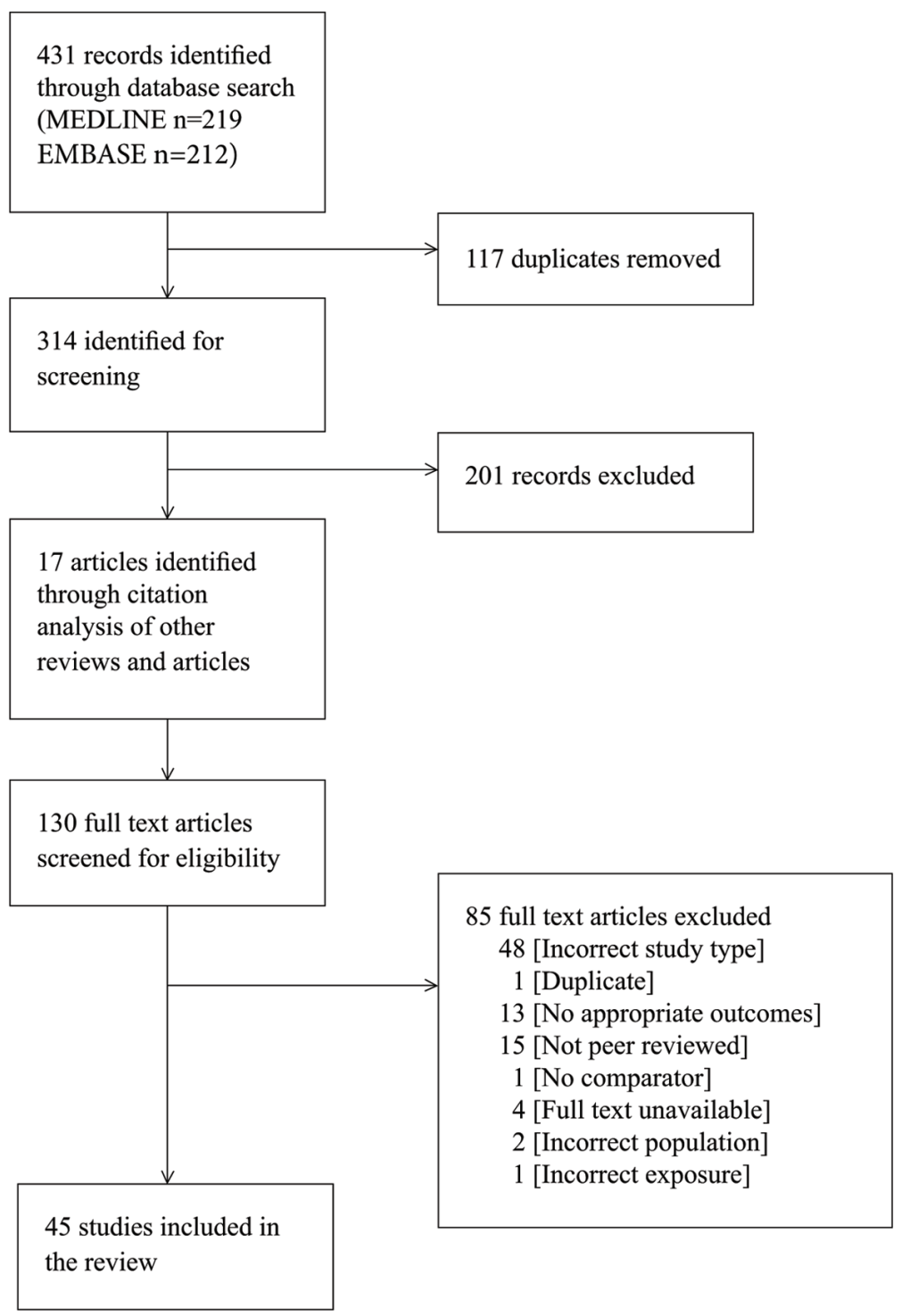

Figure 1. PRISMA flow diagram showing results of search and study selection.

was used to assess publication bias. An asymmetric graphic indicates bias. Asymmetry was tested using the Begg and Egger test [19]. Stata version 13 was used to perform the meta-analysis and statistical analysis.

\section{Results}

The literature search yielded 431 articles. After removing duplicates and excluding articles on the basis of their title, abstract, or full text (Supplementary Material 1, www.jofem. org), 45 studies $(22,091$ patients) met the inclusion criteria (Fig. 1) [2, 5, 20-62]. The majority of studies included hospitalized patients with moderate to severe COVID-19; only five studies included mild cases [42-44, 47, 48]. The majority of patients were adults, with median ages ranging from 44 to 65 . The majority of the studies were set in China $(n=40)$, with the remainder in Korea $(\mathrm{n}=1)$, USA $(\mathrm{n}=2)$, and France $(\mathrm{n}=$ 2). Enrolment dates ranged from December 11, 2019 to April 18,2020 . The majority of the studies were retrospective cohort studies, with the exception of one case-control [40] and one prospective cohort study [5]. The determination of exposure (diabetes) and comparator groups was mostly either through medical records or was self-reported. The type of diabetes was not identified in 41 studies, with those that did identify types reporting $88 \%$ of patients with diabetes as having type 2 diabetes $[5,25]$, or excluding all patients without type 2 diabetes $[21,62]$. Of these 45 studies, only 14 studies had outcome data for the primary objective (12,383 patients); these studies were summarised and presented in Table $1[2,29,33,35,38,40-42$, $46,50,58,59,61,62]$. Forty-two studies with outcome data for the secondary objective (18,878 patients) and the Table of study characteristics for the additional 31 studies as well as the Table of participant characteristics are available here (Supple- 


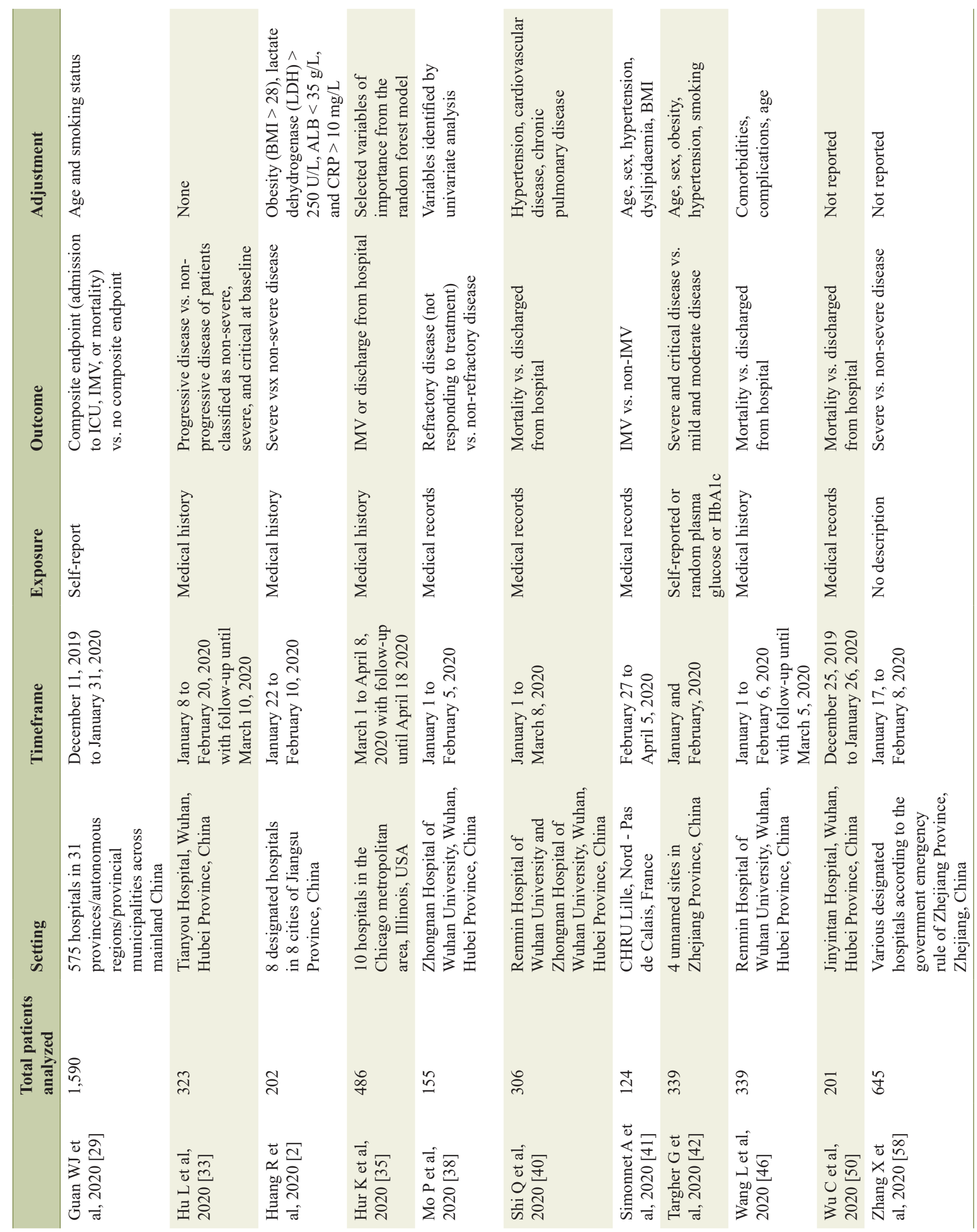




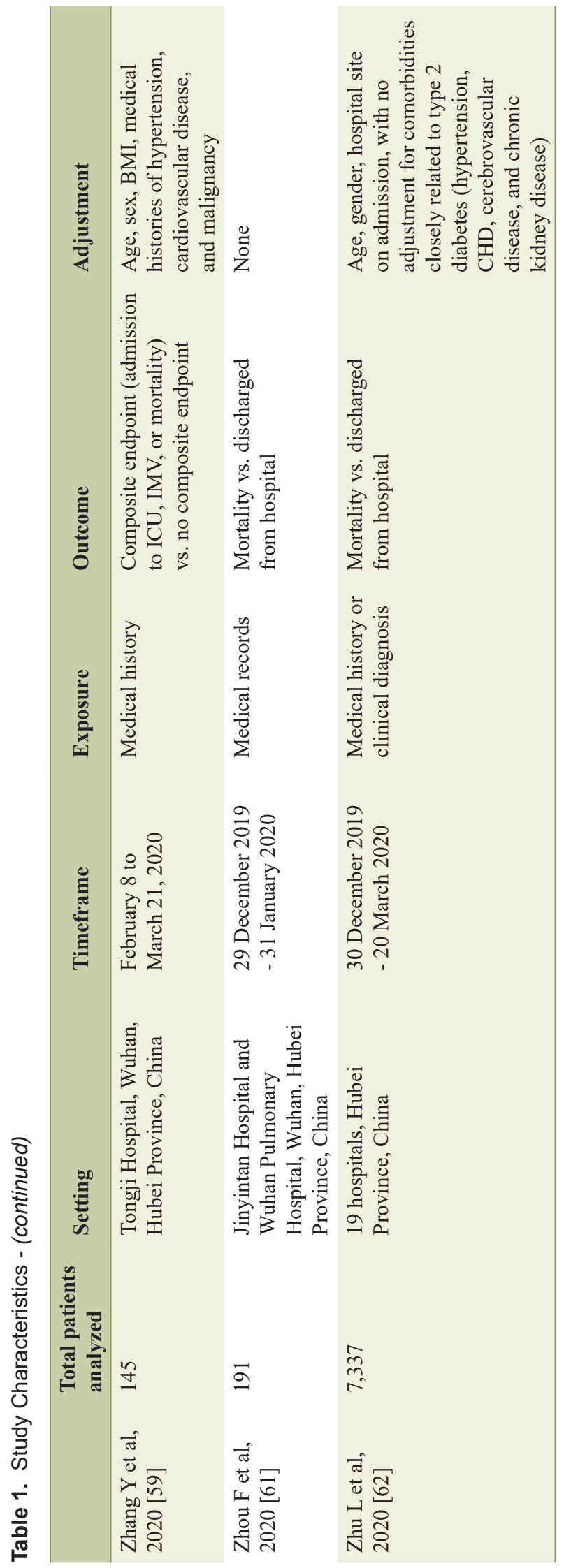

mentary Material 1, www.jofem.org).

The primary objective of the association between diabetes and the composite outcome is presented in Figure $2[15,16$, 63]. From studies that reported hazard ratios, patients with diabetes had 1.59 (95\% CI 1.3 - 1.93) times higher risk of experiencing composite outcomes than patients without diabetes; the associated level of heterogeneity between the pooled studies $(\mathrm{n}=5)$ was low $\left(\mathrm{I}^{2}=0 \%, \mathrm{P}=0.820\right)$. From the retrospective studies that reported odd ratios, patients with diabetes had 2.15 ( $95 \%$ CI 1.63 - 2.83) times increased odds of the composite outcome compared with those without diabetes, and the associated level of heterogeneity between the pooled studies ( $\mathrm{n}=$ 9) was low $\left(\mathrm{I}^{2}=0 \%, \mathrm{P}=0.892\right)$.

The primary objective pooled adjusted measures of association, as presented and adjusted by the study authors for within-study covariates (studies meeting these criteria $n=14$ ). To investigate what effect using these adjusted measures of association had on the direction or magnitude of the pooled estimate, we pooled the crude/unadjusted participant data from all studies that presented data in this $(n=42)$ without adjusting for within-study covariates (Supplementary Material 1, www. jofem.org). The crude/unadjusted odds ratio was 1.53 (95\% CI 1.43 - 1.62) with high heterogeneity $\left(\mathrm{I}^{2}=83.2 \%\right)$. In comparison with the adjusted measure of association, the direction and magnitude of the effect remained unchanged; however, the heterogeneity was high.

The secondary objective, the pooled prevalence of diabetes in COVID-19 patients experiencing composite outcome, was $24 \%$ (95\% CI $20-27 \%$ ) (Fig. 3 ). The level of heterogeneity between the studies included in this analysis was high $\left(\mathrm{I}^{2}\right.$ $=86.24 \%, \mathrm{P}=0.00)$. Therefore, a predefined sub-group analysis was performed to adjust for expected sources of heterogeneity. The studies were separated by geographical region, with those set outside of Asia reporting a prevalence of $45 \%$ (CI 23-68\%) and those set in Asia reporting a prevalence of $21 \%$ (CI 18-24\%; $\mathrm{I}^{2}=78.56, \mathrm{P}=0.00$ ) (Fig. 3). Prevalence of diabetes separated by quality of the studies reported a prevalence of $22 \%$ (CI 17-26\%; $\left.\mathrm{I}^{2}=86.64, \mathrm{P}=0.00\right)$ in those rated fair quality and a prevalence of $27 \%$ (CI $21-33 \%$; $\mathrm{I}^{2}=$ $88.17, \mathrm{P}=0.00$ ) in those rated good quality (Supplementary Material 1, www.jofem.org). Sub-group analysis by type of outcome experienced, mortality versus severe disease, reported a prevalence of $30 \%\left(\mathrm{CI} 21-39 \% ; \mathrm{I}^{2}=90.91, \mathrm{P}=0.00\right)$ versus $21 \%\left(\mathrm{CI} 17-24 \% ; \mathrm{I}^{2}=86.24, \mathrm{P}=0.00\right)$ (Supplementary Material 1, www.jofem.org). The prevalence of diabetes was sub-grouped into those studies with a median age of patients greater than age 60 , and those with a median age of patients of age 60 and below and reported as 34\% (CI 20-49\%; $\mathrm{I}^{2}=$ $95.74, \mathrm{P}=0.00)$ and $21 \%\left(\mathrm{CI} 17-24 \% ; \mathrm{I}^{2}=74.48, \mathrm{P}=0.00\right)$, respectively (Supplementary Material 1, www.jofem.org).

The risk of bias assessment of the 45 studies included found the study design and execution of 31 studies to be of fair quality, and those of 14 studies to be of good quality (see Table of risk of bias assessment (Supplementary Material 1, www.jofem.org)). The outcomes extracted for the synthesis of the primary outcome were found to be of moderate quality on the GRADE quality of evidence scale. The moderate-qualityranked evidence can be interpreted as meaning that the true effect is probably close to the estimated effect. Publication bias 
a

$\mathrm{DM}$

$\begin{array}{lll}\text { Hazard } & \% & \text { Study } \\ \text { Ratio }(95 \% \mathrm{Cl}) & \text { Weight } & \text { quality }\end{array}$

Study Setting Outcome DM/CO No CO

Ratio $(95 \% \mathrm{Cl}) \quad$ Weight quality

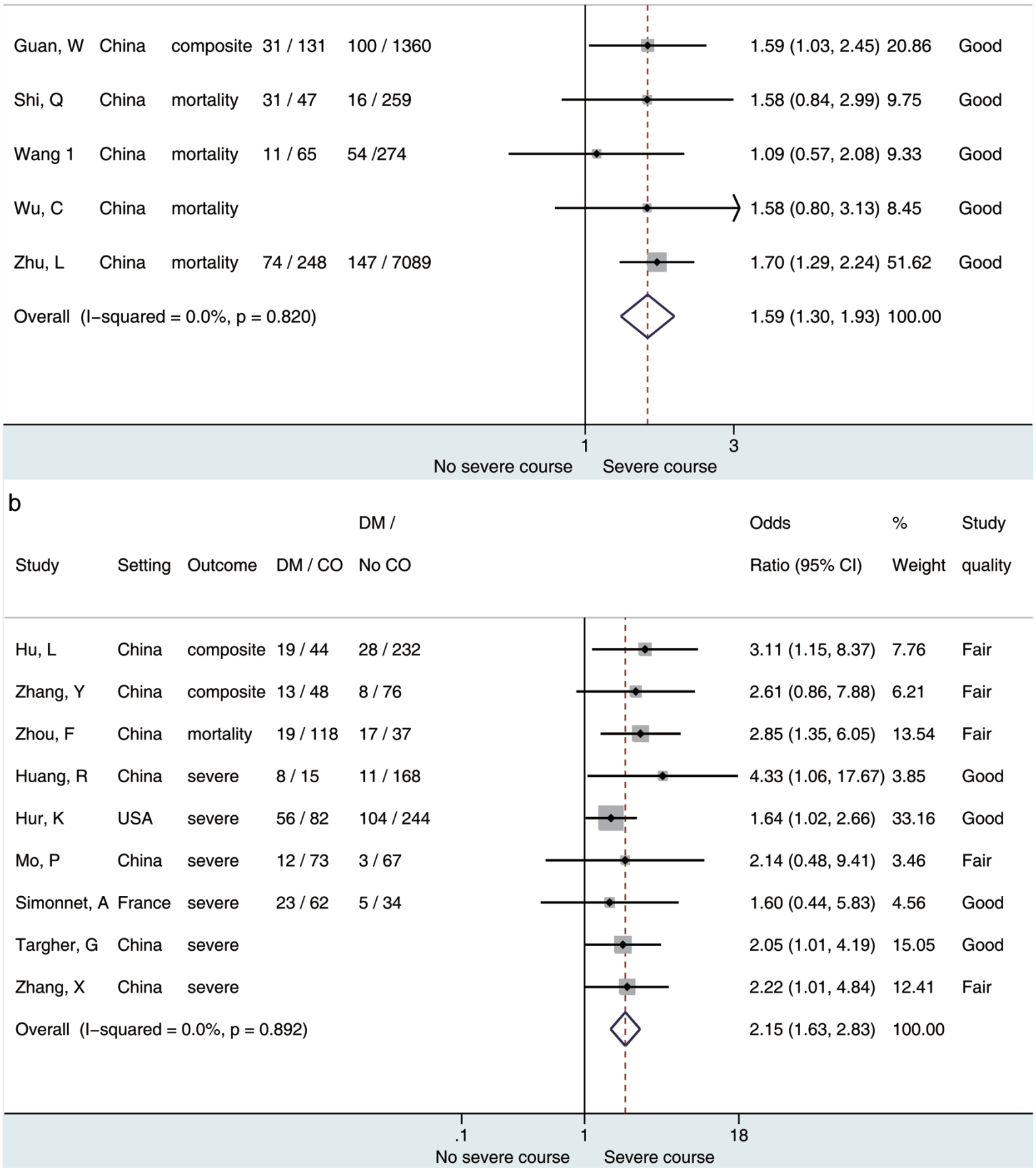

Figure 2. (a) Forest plot showing pooled hazard ratio of diabetes associated with composite outcome of patients hospitalized for COVID-19. (b) Forest plot showing pooled odds ratio of diabetes associated with composite outcome of patients hospitalized for COVID-19. Composite outcome = mortality/admittance to ICU/requirement for IMV/clinically diagnosed with refractory, progressive, severe, or critical disease [15, 16, 63]; severe outcome = any except mortality. DM: diabetes mellitus; CO: composite outcome. 


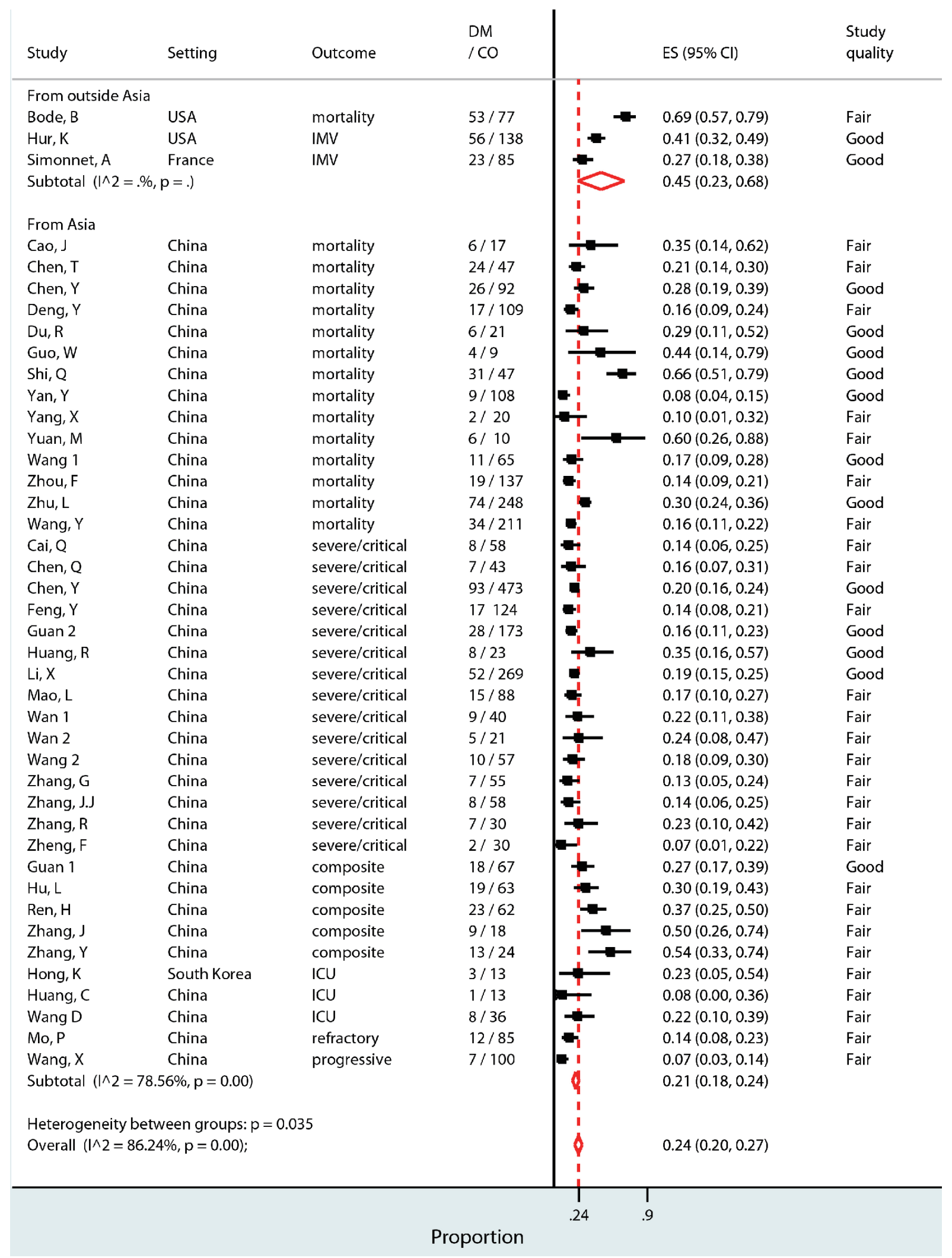

Figure 3. Pooled prevalence proportion of patients with diabetes among patients hospitalized for COVID-19 who experienced composite outcome (severe disease, admittance to ICU, requirement for IMV, or death), sub-grouped into studies set in Asia (China and Korea) and those outside of Asia (USA and France). 


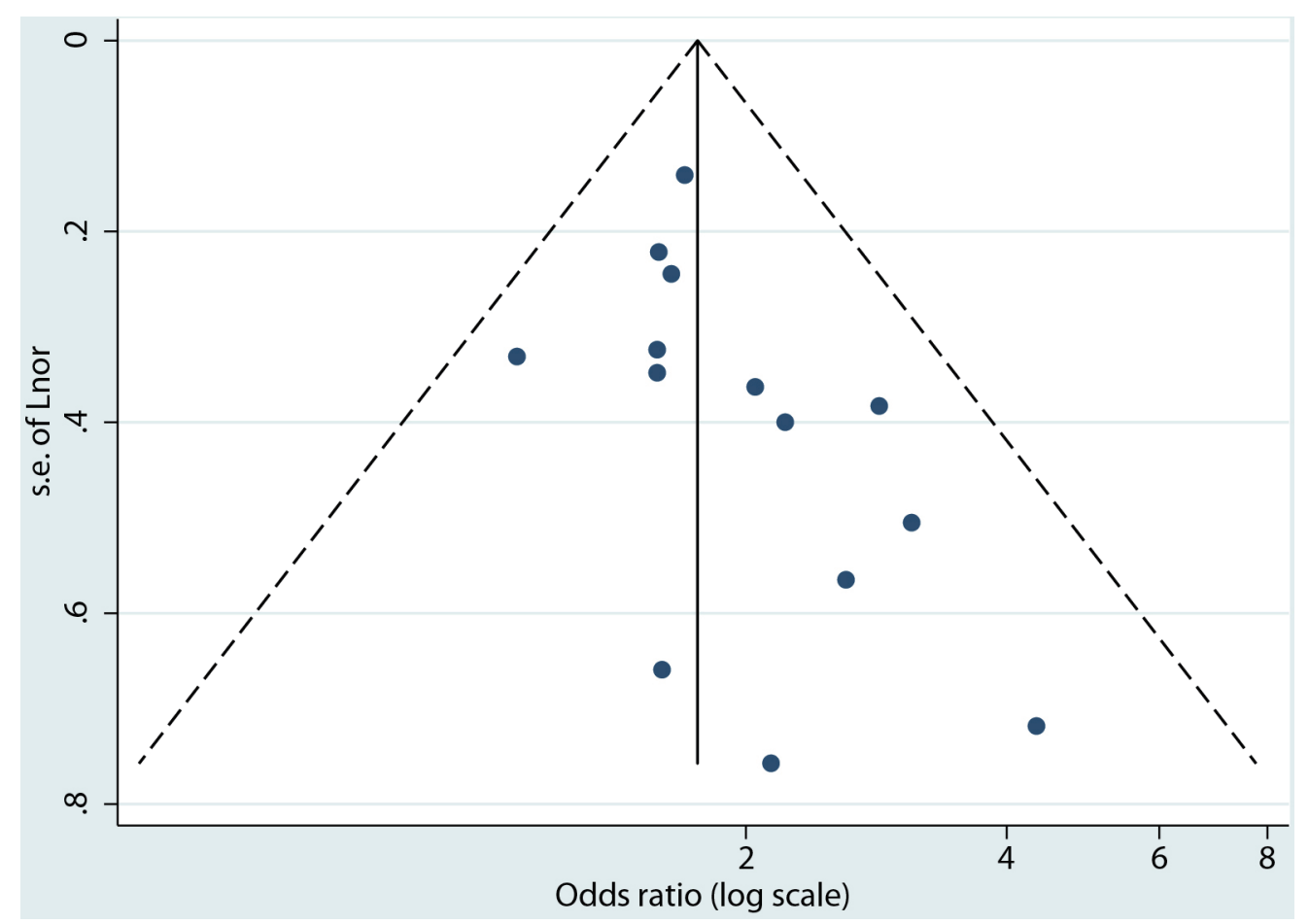

Figure 4. Funnel plot for the evaluation of publication bias.

was evaluated through the visual inspection of a funnel plot and tested using the Begg and Egger test (Fig. 4) [19]. The funnel plot was asymmetrical, indicating that there may be publication bias. However, the Begg and Egger test for asymmetry suggests that asymmetry is not significant, with $\mathrm{P}=0.077$.

We investigated the potential of overlapping study populations, as 40 of the 45 studies were from similar locations, timeframes, and authors. There was no conclusive overlap, but the review team identified 29 studies [22-24, 26-28, 30, 31, 33, $34,36-38,44-57,59,61]$ that could potentially overlap (details in Table of potentially overlapping study populations (Supple- mentary Material 1, www.jofem.org)). Removing these studies from the analysis, the primary objective was analyzed using only the six remaining eligible studies [2, 29, 35, 41, 58, 62]. The pooled measure of association from four studies $[2,35,41$, 58] was an adjusted odds ratio of 1.88 (95\% CI 1.29 - 2.74), and the pooled measure of association from two studies [29, 62] was an adjusted hazard ratio of 1.67 (95\% CI $1.32-2.10)$ (Supplementary Material 1, www.jofem.org). Therefore, even when excluding potentially overlapping study populations, the direction and magnitude of the effect remained similar, although CIs increased.

Table 2. Putative Prognostic Factors

Uncontrolled hyperglycemia

Insulin use

Triglyceride and glucose (TyG) index marker for insulin resistance
A retrospective cohort study $(\mathrm{n}=570)$ in the USA investigating blood glucose levels among hospitalized patients with diabetes for acute hyperglycemia found that there is an association. The study reported that hospitalized COVID-19 patients with diabetes and/or uncontrolled hyperglycemia had a higher prevalence of mortality as compared with patients without diabetes or uncontrolled hyperglycaemia [20]. Diabetes was defined as A1c $\geq$ $6.5 \%$ [20]. Uncontrolled hyperglycemia was defined as $\geq 2$ blood glucoses (BGs) $>180 \mathrm{mg} / \mathrm{dL}$ within any $24-\mathrm{h}$ period [20]. However, the CORONADO prospective cohort study with 1,317 COVID-19 patients with diabetes from 53 hospitals in France, where the HbA1C level of the patients was examined, did not find that long-term glycemic control impacted the severity of COVID-19 disease within the first 7 days of admission to hospital [5].

A retrospective study of patients with diabetes $(n=136)$ and those without diabetes $(n=768)$ with moderate, severe, or critical COVID-19 in Wuhan, China, noted the use of insulin in patients with diabetes was related to poor prognosis of COVID-19 clinical course [25]. However, in the CORONADO study, insulin use was not associated with a severe prognosis (intubation and/or death on day 7) in a multivariable analysis after adjustment [5]. Insulin use may be a proxy of advanced diabetes in older people with complications such as chronic kidney disease (CKD), rather than a causal factor of COVID-19 severity [5].

A retrospective study of 151 patients in Wuhan, China, who were admitted to hospital with moderate to severe COVID-19 found an increasing TyG index to predict increased odds of severe or mortal outcomes from COVID-19 [39]. 
Uncontrolled hyperglycemia, insulin use, and insulin resistance were extracted from the included studies as part of the exploratory outcome to report putative prognostic factors in patients with diabetes, who were hospitalized due to COVID-19, and were more likely to experience severe outcomes or death (Table $2[5,20,25,39])$.

\section{Discussion}

Several meta-analyses have reported on the association between severe COVID-19 and diabetes using crude data extracted from studies to obtain unadjusted odds ratios and pooled measures of association. The current rapid review and meta-analysis pools the within-study adjusted measure of association and therefore strengthens the evidence emerging from the pandemic, revealing the association between diabetes and severe clinical outcomes if people with pre-existing diabetes become hospitalized due to infection with COVID-19. In agreement with emerging evidence [6, 10, 64-67], we report that people hospitalized for COVID-19 with pre-existing diabetes have a 1.6 times increased risk or 2-fold increased odds of experiencing the composite outcome (mortality, admittance to ICU, requirement for IMV, clinically defined severe or critical disease). This finding strengthens the evidence of diabetes as a risk factor in patients hospitalized due to COVID-19 and their increased likelihood of developing a severe clinical course.

As with other similar meta-analyses conducted at this stage of the pandemic, the majority of studies are from China $[10,64,66,68]$. We report the pooled prevalence of diabetes in COVID-19 patients who experienced the composite outcome as $24 \%$ (95\% CI $20-27 \%$ ). We found that studies, mostly in China, reported a diabetes prevalence in hospitalized COVID-19 patients with severe clinical course of $21 \%$ (95\% CI 18 $24 \%$ ), while in settings outside of China the prevalence was $45 \%$ (95\% CI 23-68\%). However, the CIs were large, there were few studies outside of Asia, and there was a high level of heterogeneity, therefore this finding should be interpreted with caution. Indeed, global distribution of prevalence of diabetes varies across regions, with China's prevalence of diabetes in adults estimated at about $8.8 \%$, whereas a significantly higher prevalence of diabetes is observed in other regions of the world where COVID-19 is now present [7]. This potentially indicates that the burden of a severe COVID-19 clinical course may be greater in countries outside of Asia with a higher prevalence of diabetes than those reported thus far in the pandemic. For instance, in North America, which has a diabetes prevalence of $13 \%$ [7], it was recently reported that mortality in patients with COVID-19 was 12 times higher among those with underlying conditions such as diabetes and cardiovascular disease [69]. As the epicentre of the pandemic shifts from China to Europe, the USA, and the rest of the world, there is a definite need for reviews such as this one to continue updating the current evidence base to reflect the present reality of the pandemic and ensure the best possible care for people, who are at higher risk, especially in countries with a population with a high prevalence of diabetes.
One strength of our meta-analysis is an analysis approach designed to ensure quality. To this end, we avoided publications that had not been subjected to external peer review, we investigated the potential effect of population overlap on the direction and magnitude of the effect, and thirdly we extracted and pooled measures of association with within-study adjustments for covariates for the primary objective. The measures of association adjusted for common covariates within studies, including age, sex, and comorbidities, that are not accounted for when using a simple pooling method of crude/unadjusted measures of association, thereby addressing a common issue that can arise as a result of the simple pooling approach [70]. We only pooled measures of association from analytical study designs, not from descriptive study designs, to ensure the outcomes reported are more likely to represent the patient's clinical course without missing potential severe outcomes or death due to follow-up not being of sufficient duration and the patient still being in hospital at the end of the study period.

One of the benefits of the rapid review approach during the pandemic is the ability to identify key evidence gaps that require further investigation and understanding in order to answer critical, clinically relevant questions. An evidence gap identified is the need to identify prognostic risk factors in people living with diabetes who are more likely to go on to experience severe clinical course. The identification of prognostic factors would allow for prioritizing earlier dedication of scarce resources and better risk management. The exploratory objective of this review identified glycemic control prior to hospital admission as a putative prognostic factor that could be included in future research [20]. While recognizing that we do not have the strength of evidence to suggest glycemic control is a risk factor [5], we suggest that clinicians should focus on good glycemic control in their patients who have diabetes, as this might benefit them should they become infected with COVID-19. Moreover, concordant studies identified admission blood glucose level as a major predictor of severe COVID-19 outcomes in patients with and also without known diabetes [5, 59, 62]. Dedicated randomized controlled trials are warranted to determine whether tight glycemic control during hospitalisation can improve COVID-19 prognosis.

\section{Limitations}

This study has several limitations related to the observational nature of the studies reviewed, including the uncertainty of participants being assigned to exposure or comparator groups. In addition, the study was limited to hospitalized patients and therefore represents the moderate to critical spectrum of $\mathrm{COV}$ ID-19 disease. We also found that the majority of the studies did not include information on body mass index (BMI) or HbAlc before hospitalization. In addition, it is accepted that retrospective studies make it hard to collect information on glycemic variability or hypoglycemic treatments during hospitalization (in a usual scenario of hyperglycemia associated with cytokine storm and/or glucocorticoid treatment). Furthermore, there was a lack of information on type 1 diabetes and the association with severe COVID-19 clinical course. There was also a lack of information from studies located outside 
of China. In addition, there is the potential overlap of study populations, especially those in China that enrolled at the same time in the same area of Wuhan in Hubei Province. The study authors of the largest study [62] were contacted twice, but with no response. As this is a rapid review with the intention of providing timely information for decision-makers, we have proceeded without their input.

\section{Conclusions}

In summary, this rapid review identifies that people living with diabetes are more likely to develop severe COVID-19 clinical course if hospitalized for COVID-19 than people not living with diabetes. To inform clinical decision-making during the pandemic, our findings support that people living with diabetes who are hospitalized for COVID-19 should be prioritized when triaged as at increased risk for the development of severe clinical course. Clinicians, policymakers, and decision-makers urgently need to be aware that people living with diabetes, if they become hospitalized due to COVID-19, are at increased risk of developing COVID-19 severe clinical course (admittance to ICU for specialized treatment, requirement for IMV, clinically defined severe or critical disease, and/or death). Further research is needed to strengthen the finding of increased risk of diabetes with severe COVID-19 outcomes, especially outside of Asia, and to determine whether these findings also apply to people living with type 1 diabetes. In addition, the identification of prognostic factors in people living with diabetes who are hospitalized for COVID-19 and who develop severe COVID-19 disease would be hugely valuable in assessing risk within the population of COVID-19 hospitalized diabetes patients.

\section{Supplementary Material}

Suppl 1. Supplementary information.

\section{Acknowledgments}

Craig Ludwig, managing director of Last Mile, provided project management support and critical feedback on the draft rapid review. David Reeves, an information specialist and independent consultant, performed the search strategy as well as the screening of studies. Paul Otiku, a methodologist and independent consultant, performed the screening, risk of bias, and GRADE analysis.

\section{Financial Disclosure}

This study was sponsored by Novo Nordisk. The sponsor (Novo Nordisk) reviewed the protocol, study design and manuscript for medical and scientific accuracy ahead of author approval for submission. The sponsor had no role in the data collection. The authors interpreted the data and wrote the manuscript with the help of medical writing services funded by the sponsor. The corresponding author had full access to all data in the study and had final responsibility for the decision to submit for publication.

\section{Conflict of Interest}

HM reports personal fees from Last Mile, and outside the submitted work, HM is part of the Evidence-Based Healthcare and Public Health in Africa (CEBHA+) Scholarship Programme. $\mathrm{CEBHA}+$ receives funding from the Federal Ministry for Education and Research (Bundesministerium fur Bildung und Forschung, BMBF), Germany. MK reports personal fees from Last Mile, and outside the submitted work, MK is part of the HIV-associated Tuberculosis Training Program Fogarty Fellowship supported by the National Institutes of Health. NM is employed by Last Mile P/S. Last Mile receives consultancy fees from Novo Nordisk. NH, UHP, and CH are employed by Novo Nordisk. BC reports grants and personal fees from Amgen, Astra-Zeneca, Akcea, Genfit, Gilead, Eli Lilly, Novo Nordisk, and Merck (MSD), and grants and personal fees from Sanofi and Regeneron, outside the submitted work. MAR reports grants and personal fees from Novo Nordisk, and personal fees from AstraZeneca, Eli Lilly, Janssen, MSD, Mundipharma, and Sanofi.

\section{Informed Consent}

Not applicable.

\section{Author Contributions}

HM designed the study with input from all authors $(\mathrm{BC}, \mathrm{CH}$, MAR, MK, NH, NM, UHP). HM and NM contributed to study selection with support from two independent consultants. HM planned the statistical analysis with $\mathrm{MK}$, and extracted the data with review of data extraction from a second reviewer. HM and MK analyzed data. HM independently performed ROB analysis with a second reviewer. HM reviewed the quality analysis perfomed independently by another consultant. HM wrote the manuscript with assistance with the writing the first draft from MK and editorial assistance from NM. All authors contributed to data interpretation, writing of the discussion, as well critical review of the manuscript (BC, $\mathrm{CH}, \mathrm{HM}, \mathrm{MAR}, \mathrm{MK}, \mathrm{NH}, \mathrm{NM}$, $\mathrm{UHP})$. NM is the guarantor.

\section{Data Availability}

No additional data available. All data relevant to the study are included in the article or uploaded as supplementary information.

\section{References}

1. Mahase E. Covid-19: WHO declares pandemic because 
of "alarming levels" of spread, severity, and inaction. BMJ. 2020;368:m1036.

2. Huang R, Zhu L, Xue L, Liu L, Yan X, Wang J, Zhang B, et al. Clinical findings of patients with coronavirus disease 2019 in Jiangsu province, China: A retrospective, multicenter study. PLoS Negl Trop Dis. 2020;14(5):e0008280.

3. Nishiura H, Kobayashi T, Miyama T, Suzuki A, Jung SM, Hayashi K, Kinoshita R, et al. Estimation of the asymptomatic ratio of novel coronavirus infections (COVID-19). Int J Infect Dis. 2020;94:154-155.

4. CDC COVID-19 Response Team. Severe outcomes among patients with coronavirus disease 2019 (COVID-19) - United States, February 12-March 16, 2020. MMWR Morb Mortal Wkly Rep. 2020;69(12):343-346.

5. Cariou B, Hadjadj S, Wargny M, Pichelin M, Al-Salameh A, Allix I, Amadou C, et al. Phenotypic characteristics and prognosis of inpatients with COVID-19 and diabetes: the CORONADO study. Diabetologia. 2020;63(8):15001515 .

6. Huang I, Lim MA, Pranata R. Diabetes mellitus is associated with increased mortality and severity of disease in COVID-19 pneumonia - A systematic review, metaanalysis, and meta-regression. Diabetes Metab Syndr. 2020;14(4):395-403.

7. International Diabetes Federation. IDF Diabetes Atlas, 9th edn. Brussels, Belgium; 2019.

8. van Crevel R, van de Vijver S, Moore DAJ. The global diabetes epidemic: what does it mean for infectious diseases in tropical countries? Lancet Diabetes Endocrinol. 2017;5(6):457-468.

9. Espinosa OA, Zanetti ADS, Antunes EF, Longhi FG, Matos TA, Battaglini PF. Prevalence of comorbidities in patients and mortality cases affected by SARS-CoV2: a systematic review and meta-analysis. Rev Inst Med Trop Sao Paulo. 2020;62:e43.

10. Kumar A, Arora A, Sharma P, Anikhindi SA, Bansal N, Singla V, Khare $\mathrm{S}$, et al. Is diabetes mellitus associated with mortality and severity of COVID-19? A meta-analysis. Diabetes Metab Syndr. 2020;14(4):535-545.

11. Moher D, Liberati A, Tetzlaff J, Altman DG, The PRISMA Group. Preferred reporting items for systematic reviews and meta-analyses: the PRISMA statement. J Clin Epidemiol. 2009;62(10):1006-1012.

12. Ovid Expert Searches [Internet]. 2020. Available from: https://tools.ovid.com/ovidtools/expertsearches. html\#corona.

13. Mathes T, Pieper D. Clarifying the distinction between case series and cohort studies in systematic reviews of comparative studies: potential impact on body of evidence and workload. BMC Med Res Methodol. 2017;17(1):107.

14. World Health Organization. Report of the WHO-China Joint Mission on coronavirus disease 2019 (COVID-19). 2020.

15. National Health Commission of the People's Republic of China. Diagnosis and treatment protocol for COVID-19 (trial version 7). 2020.

16. Metlay JP, Waterer GW, Long AC, Anzueto A, Brozek J, Crothers K, Cooley LA, et al. Diagnosis and treatment of adults with community-acquired pneumonia. An official clinical practice guideline of the American Thoracic Society and Infectious Diseases Society of America. Am J Respir Crit Care Med. 2019;200(7):e45-e67.

17. DerSimonian R, Laird N. Meta-analysis in clinical trials. Control Clin Trials. 1986;7(3):177-188.

18. Wells G SB, O'Connell D, Peterson J, Welch V, Losos M, Tugwell P. The Newcastle-Ottawa Scale (NOS) for assessing the quality of nonrandomised studies in metaanalyses 2013 [May 21, 2020]. Available from: http:// www.ohri.ca/programs/clinical_epidemiology/oxford. asp.

19. Egger M, Davey Smith G, Schneider M, Minder C. Bias in meta-analysis detected by a simple, graphical test. BMJ. 1997;315(7109):629-634.

20. Bode B, Garrett V, Messler J, McFarland R, Crowe J, Booth R, Klonoff DC. Glycemic characteristics and clinical outcomes of COVID-19 patients hospitalized in the United States. J Diabetes Sci Technol. 2020;14(4):813821.

21. Cai Q, Huang D, Ou P, Yu H, Zhu Z, Xia Z, Su Y, et al. COVID-19 in a designated infectious diseases hospital outside Hubei Province, China. Allergy. 2020;75(7):17421752.

22. Cao J, Tu WJ, Cheng W, Yu L, Liu YK, Hu X, Liu Q. Clinical features and short-term outcomes of 102 patients with coronavirus disease 2019 in Wuhan, China. Clin Infect Dis. 2020;71(15):748-755.

23. Chen Q, Zheng Z, Zhang C, Zhang X, Wu H, Wang J, Wang S, et al. Clinical characteristics of 145 patients with corona virus disease 2019 (COVID-19) in Taizhou, Zhejiang, China. Infection. 2020;48(4):543-551.

24. Chen T, Wu D, Chen H, Yan W, Yang D, Chen G, Ma $\mathrm{K}$, et al. Clinical characteristics of 113 deceased patients with coronavirus disease 2019: retrospective study. BMJ. 2020;368:m1091.

25. Chen Y, Yang D, Cheng B, Chen J, Peng A, Yang C, Liu $\mathrm{C}$, et al. Clinical characteristics and outcomes of patients with diabetes and COVID-19 in association with glucoselowering medication. Diabetes Care. 2020;43(7):13991407.

26. Deng Y, Liu W, Liu K, Fang YY, Shang J, Zhou L, Wang $\mathrm{K}$, et al. Clinical characteristics of fatal and recovered cases of coronavirus disease 2019 in Wuhan, China: a retrospective study. Chin Med J (Engl). 2020;133(11):12611267.

27. Du RH, Liang LR, Yang CQ, Wang W, Cao TZ, Li M, Guo GY, et al. Predictors of mortality for patients with COVID-19 pneumonia caused by SARS-CoV-2: a prospective cohort study. Eur Respir J. 2020;55(5).

28. Feng Y, Ling Y, Bai T, Xie Y, Huang J, Li J, et al. COVID-19 with different severity: a multi-center study of clinical features. Am J Respir Crit Care Med. 2020;201(11):1380-1388.

29. Guan WJ, Liang WH, Zhao Y, Liang HR, Chen ZS, Li YM, Liu XQ, et al. Comorbidity and its impact on 1590 patients with COVID-19 in China: a nationwide analysis. Eur Respir J. 2020;55(5).

30. Guan WJ, Ni ZY, Hu Y, Liang WH, Ou CQ, He JX, Liu L, 
et al. Clinical characteristics of coronavirus disease 2019 in China. N Engl J Med. 2020;382(18):1708-1720.

31. Guo W, Li M, Dong Y, Zhou H, Zhang Z, Tian C, Qin $\mathrm{R}$, et al. Diabetes is a risk factor for the progression and prognosis of COVID-19. Diabetes Metab Res Rev. 2020:e3319.

32. Hong KS, Lee KH, Chung JH, Shin KC, Choi EY, Jin HJ, Jang JG, et al. Clinical features and outcomes of 98 patients hospitalized with SARS-CoV-2 infection in Daegu, South Korea: a brief descriptive study. Yonsei Med J. 2020;61(5):431-437.

33. Hu L, Chen S, Fu Y, Gao Z, Long H, Wang JM, Ren HW, et al. Risk factors associated with clinical outcomes in 323 COVID-19 hospitalized patients in Wuhan, China. Clin Infect Dis. 2020.

34. Huang C, Wang Y, Li X, Ren L, Zhao J, Hu Y, Zhang $\mathrm{L}$, et al. Clinical features of patients infected with 2019 novel coronavirus in Wuhan, China. Lancet. 2020;395(10223):497-506.

35. Hur K, Price CPE, Gray EL, Gulati RK, Maksimoski M, Racette SD, Schneider AL, et al. Factors associated with intubation and prolonged intubation in hospitalized patients with COVID-19. Otolaryngol Head Neck Surg. 2020;163(1):170-178.

36. Li X, Xu S, Yu M, Wang K, Tao Y, Zhou Y, Shi J, et al. Risk factors for severity and mortality in adult COVID-19 inpatients in Wuhan. J Allergy Clin Immunol. 2020;146(1):110-118.

37. Mao L, Jin H, Wang M, Hu Y, Chen S, He Q, Chang J, et al. Neurologic manifestations of hospitalized patients with coronavirus disease 2019 in Wuhan, China. JAMA Neurol. 2020;77(6):683-690.

38. Mo P, Xing Y, Xiao Y, Deng L, Zhao Q, Wang H, Xiong $\mathrm{Y}$, et al. Clinical characteristics of refractory COVID-19 pneumonia in Wuhan, China. Clin Infect Dis. 2020.

39. Ren H, Yang Y, Wang F, Yan Y, Shi X, Dong K, Yu X, et al. Association of the insulin resistance marker TyG index with the severity and mortality of COVID-19. Cardiovasc Diabetol. 2020;19(1):58.

40. Shi Q, Zhang X, Jiang F, Zhang X, Hu N, Bimu C, Feng $\mathrm{J}$, et al. Clinical characteristics and risk factors for mortality of COVID-19 patients with diabetes in Wuhan, China: a two-center, retrospective study. Diabetes Care. 2020;43(7):1382-1391.

41. Simonnet A, Chetboun M, Poissy J, Raverdy V, Noulette J, Duhamel A, Labreuche J, et al. High prevalence of obesity in severe acute respiratory syndrome coronavirus-2 (SARS-CoV-2) requiring invasive mechanical ventilation. Obesity (Silver Spring). 2020;28(7):1195-1199.

42. Targher G, Mantovani A, Wang XB, Yan HD, Sun QF, Pan KH, Byrne CD, et al. Patients with diabetes are at higher risk for severe illness from COVID-19. Diabetes Metab. 2020;46(4):335-337.

43. Wan S, Xiang Y, Fang W, Zheng Y, Li B, Hu Y, Lang C, et al. Clinical features and treatment of COVID-19 patients in northeast Chongqing. J Med Virol. 2020;92(7):797806.

44. Wan S, Yi Q, Fan S, Lv J, Zhang X, Guo L, Lang C, et al. Relationships among lymphocyte subsets, cy- tokines, and the pulmonary inflammation index in coronavirus (COVID-19) infected patients. Br J Haematol. 2020;189(3):428-437.

45. Wang D, Hu B, Hu C, Zhu F, Liu X, Zhang J, Wang B, et al. Clinical characteristics of 138 hospitalized patients with 2019 novel coronavirus-infected pneumonia in $\mathrm{Wu}-$ han, China. JAMA. 2020;323(11):1061-1069.

46. Wang L, He W, Yu X, Hu D, Bao M, Liu H, Zhou J, et al. Coronavirus disease 2019 in elderly patients: characteristics and prognostic factors based on 4-week follow-up. J Infect. 2020;80(6):639-645.

47. Wang L, Li X, Chen H, Yan S, Li D, Li Y, Gong Z. Coronavirus disease 19 infection does not result in acute kidney injury: an analysis of 116 hospitalized patients from Wuhan, China. Am J Nephrol. 2020;51(5):343-348.

48. Wang X, Fang J, Zhu Y, Chen L, Ding F, Zhou R, Ge L, et al. Clinical characteristics of non-critically ill patients with novel coronavirus infection (COVID-19) in a Fangcang Hospital. Clin Microbiol Infect. 2020;26(8):10631068.

49. Wang Y, Lu X, Li Y, Chen H, Chen T, Su N, Huang F, et al. Clinical course and outcomes of 344 intensive care patients with COVID-19. Am J Respir Crit Care Med. 2020;201(11):1430-1434.

50. Wu C, Chen X, Cai Y, Xia J, Zhou X, Xu S, Huang H, et al. Risk factors associated with acute respiratory distress syndrome and death in patients with coronavirus disease 2019 pneumonia in Wuhan, China. JAMA Intern Med. 2020;180(7):934-943.

51. Yan Y, Yang Y, Wang F, Ren H, Zhang S, Shi X, Yu X, et al. Clinical characteristics and outcomes of patients with severe covid-19 with diabetes. BMJ Open Diabetes Res Care. 2020;8(1).

52. Yang X, Yu Y, Xu J, Shu H, Xia J, Liu H, Wu Y, et al. Clinical course and outcomes of critically ill patients with SARS-CoV-2 pneumonia in Wuhan, China: a single-centered, retrospective, observational study. Lancet Respir Med. 2020;8(5):475-481.

53. Yuan M, Yin W, Tao Z, Tan W, Hu Y. Association of radiologic findings with mortality of patients infected with 2019 novel coronavirus in Wuhan, China. PLoS One. 2020;15(3): 0230548.

54. Zhang G, Hu C, Luo L, Fang F, Chen Y, Li J, Peng Z, et al. Clinical features and short-term outcomes of 221 patients with COVID-19 in Wuhan, China. J Clin Virol. 2020;127:104364.

55. Zhang J, Yu M, Tong S, Liu LY, Tang LV. Predictive factors for disease progression in hospitalized patients with coronavirus disease 2019 in Wuhan, China. J Clin Virol. 2020;127:104392.

56. Zhang JJ, Dong X, Cao YY, Yuan YD, Yang YB, Yan YQ, Akdis CA, et al. Clinical characteristics of 140 patients infected with SARS-CoV-2 in Wuhan, China. Allergy. 2020;75(7):1730-1741.

57. Zhang R, Ouyang H, Fu L, Wang S, Han J, Huang K, Jia M, et al. CT features of SARS-CoV-2 pneumonia according to clinical presentation: a retrospective analysis of 120 consecutive patients from Wuhan city. Eur Radiol. 2020;30(8):4417-4426. 
58. Zhang X, Cai H, Hu J, Lian J, Gu J, Zhang S, Ye C, et al. Epidemiological, clinical characteristics of cases of SARS-CoV-2 infection with abnormal imaging findings. Int J Infect Dis. 2020;94:81-87.

59. Zhang Y, Li H, Zhang J, Cao Y, Zhao X, Yu N, Gao Y, et al. The clinical characteristics and outcomes of patients with diabetes and secondary hyperglycaemia with coronavirus disease 2019: A single-centre, retrospective, observational study in Wuhan. Diabetes Obes Metab. 2020;22(8):1443-1454.

60. Zheng F, Tang W, Li H, Huang YX, Xie YL, Zhou ZG. Clinical characteristics of 161 cases of corona virus disease 2019 (COVID-19) in Changsha. Eur Rev Med Pharmacol Sci. 2020;24(6):3404-3410.

61. Zhou F, Yu T, Du R, Fan G, Liu Y, Liu Z, Xiang J, et al. Clinical course and risk factors for mortality of adult inpatients with COVID-19 in Wuhan, China: a retrospective cohort study. Lancet. 2020;395(10229):1054-1062.

62. Zhu L, She ZG, Cheng X, Qin JJ, Zhang XJ, Cai J, Lei F, et al. Association of Blood Glucose Control and Outcomes in Patients with COVID-19 and Pre-existing Type 2 Diabetes. Cell Metab. 2020;31(6):1068-1077 e1063.

63. World Health Organization. Coronavirus disease 2019 (COVID-19) situation report - 51. 2020. March 11, 2020.
64. Wu ZH, Tang Y, Cheng Q. Diabetes increases the mortality of patients with COVID-19: a meta-analysis. Acta Diabetol. 2020.

65. Mantovani A, Byrne CD, Zheng MH, Targher G. Diabetes as a risk factor for greater COVID-19 severity and inhospital death: A meta-analysis of observational studies. Nutr Metab Cardiovasc Dis. 2020;30(8):1236-1248.

66. Roncon L, Zuin M, Rigatelli G, Zuliani G. Diabetic patients with COVID-19 infection are at higher risk of ICU admission and poor short-term outcome. J Clin Virol. 2020;127:104354.

67. Parveen R, Sehar N, Bajpai R, Agarwal NB. Association of diabetes and hypertension with disease severity in covid-19 patients: A systematic literature review and exploratory meta-analysis. Diabetes Res Clin Pract. 2020;166:108295.

68. Fadini GP, Morieri ML, Longato E, Avogaro A. Prevalence and impact of diabetes among people infected with SARS-CoV-2. J Endocrinol Invest. 2020;43(6):867-869.

69. Centers for Disease Control and Prevention. Coronavirus diesases 2019 case surveillance - United States, January 22 - May 30, 2020. United States; 2020.

70. Bravata DM, Olkin I. Simple pooling versus combining in meta-analysis. Eval Health Prof. 2001;24(2):218-230. 